\title{
Expert system-based approach to automate the design process of power electronics converters
}

\begin{abstract}
The design of power electronics converters is still an ad-hoc process. Usually, this process is accomplished virtually using existing simulation packages before the real implementation. Despite, the use of these packages, the process is proved to be time consuming and bulky task. This paper describes an expert system approach to ease this cumbersome process by automating the design procedure. The present approach combines expert system techniques with object-oriented paradigm. The designer has firstly to choose the appropriate application of the converter. Then the system suggests the most appropriate (according to the user's specifications) topology that is formed in a schematic file accepted by the Pspice simulation package. The topology is displayed within the Schematic environment containing all the circuit parameters and components including the best (optimum) switching devices and control circuits, here the simulation is launched automatically in order to investigate the outputs. The topologies constitute the knowledge base of the system represented as objects. The switching devices are stored in database module accessed by the inference engine to select the optimum switch for a certain topology. This approach could be a kernel of a power electronics converters design aid tool, whereby the complexity of the design process can be reduced dramatically.
\end{abstract}

Keyword: Class; Converter; Database; Expert system; Inference engine; Knowledge base; OOP; Switching device 